\title{
The impact of storage conditions on human stool 16S rRNA microbiome composition and diversity
}

\author{
Lauren V Carruthers ${ }^{\text {Corresp., 1, } 2 \text {, Arinaitwe Moses }}{ }^{3}$, Moses Adriko ${ }^{3}$, Christina L Faust ${ }^{1,2}{ }^{2}$, Edridah M Tukahebwa ${ }^{3}$, \\ Lindsay J Hall ${ }^{\text {Equal first author, } 4}$, Lisa C Ranford-Cartwright ${ }^{\text {Equal first author, 1 }}{ }^{\text {, Poppy H L Lamberton }}{ }^{\text {Equal first author, } 1,2}$ \\ 1 Institute of Biodiversity Animal Health and Comparative Medicine, University of Glasgow, Glasgow, United Kingdom \\ 2 Wellcome Centre for Integrative Parasitology, University of Glasgow, Glasgow, United Kingdom \\ 3 Vector Control Divison, Ugandan Ministry of Health, Kampala, Uganda \\ 4 Gut Microbes \& Health, Quadram Institute Bioscience, Norwich, United Kingdom \\ Corresponding Author: Lauren V Carruthers \\ Email address: I.carruthers.1@research.gla.ac.uk
}

Background. Multiple factors can influence stool sample integrity upon sample collection. Preservation of faecal samples for microbiome studies is therefore an important step, particularly in tropical regions where resources are limited and high temperatures may significantly influence microbiota profiles. Freezing is the accepted standard to preserve faecal samples however, cold chain methods are often unfeasible in fieldwork scenarios particularly in low and middle-income countries and alternatives are required. This study therefore aimed to address the impact of different preservative methods, time-to-freezing at ambient tropical temperatures, and stool heterogeneity on stool microbiome diversity and composition under real-life physical environments found in resource-limited fieldwork conditions. Methods. Inner, outer and mixed stool samples collected from one specimen obtained from three children were stored using different storage preservation methods (raw, ethanol and RNAlater) in a Ugandan field setting. Mixed stool was also stored using these techniques and frozen at different time-to-freezing intervals post-collection from 0 $32 \mathrm{~h}$. Metataxonomic profiling was used to profile samples, targeting the V1 - V2 regions of $16 S$ rRNA with samples run on a MiSeq platform. Reads were trimmed, combined and aligned to the Greengenes database. Microbial diversity and composition data were generated and analysed using Quantitative Insights Into Microbial Ecology (QIIME) and R software. Results. Child donor was the greatest predictor of microbiome variation between the stool samples, with all samples remaining identifiable to their child of origin despite the stool being stored under a variety of conditions. However, significant differences were observed in composition and diversity between preservation techniques, but intra-preservation technique variation was minimal for all preservation methods, and across the time-to-freezing range $(0-32 \mathrm{~h})$ used. Stool heterogeneity yielded no apparent microbiome differences. Conclusions. Stool collected in a fieldwork setting for 
comparative microbiome analyses should ideally be stored as consistently as possible using the same preservation method throughout. 
1 The impact of storage conditions on human stool $16 \mathrm{~S}$

2 rRNA microbiome composition and diversity.

3

4

\author{
Lauren V Carruthers ${ }^{1,2}$, Arinaitwe Moses $^{3}$, Moses Adriko ${ }^{3}$, Christina L Faust ${ }^{1,2}$, Edridah M \\ Tukahebwa $^{3}$, Lindsay J Hall ${ }^{1 \#}$, Lisa C Ranford-Cartwright ${ }^{1 \#}$, Poppy H L Lamberton ${ }^{1,2 \#}$ \\ ${ }^{1}$ Institute of Biodiversity Animal Health and Comparative Medicine, University of Glasgow, \\ Glasgow, UK. \\ ${ }^{2}$ Wellcome Centre for Integrative Parasitology, University of Glasgow, Glasgow, UK. \\ ${ }^{3}$ Vector Control Division, Ugandan Ministry of Health, Kampala, Uganda. \\ ${ }^{4}$ Gut Microbes \& Health, Quadram Institute Bioscience, Norwich, UK. \\ \# Contributed equally. \\ Corresponding Author: \\ Lauren V Carruthers ${ }^{1,2}$ \\ Graham Kerr Building, Institute of Biodiversity, Animal Health and Comparative Medicine, \\ University of Glasgow, Glasgow, Scotland, G12 8QQ. \\ Email address: 1.carruthers.1@research.gla.ac.uk
}

\begin{abstract}
Background. Multiple factors can influence stool sample integrity upon sample collection. Preservation of faecal samples for microbiome studies is therefore an important step, particularly in tropical regions where resources are limited and high temperatures may significantly influence microbiota profiles. Freezing is the accepted standard to preserve faecal samples however, cold chain methods are often unfeasible in fieldwork scenarios particularly in low and middle-income countries and alternatives are required. This study therefore aimed to address the impact of different preservative methods, time-to-freezing at ambient tropical temperatures, and stool heterogeneity on stool microbiome diversity and composition under real-life physical environments found in resource-limited fieldwork conditions.
\end{abstract}

Methods. Inner, outer and mixed stool samples collected from one specimen obtained from three children were stored using different storage preservation methods (raw, ethanol and RNAlater) in a Ugandan field setting. Mixed stool was also stored using these techniques and frozen at different time-to-freezing intervals post-collection from $0-32 \mathrm{~h}$. Metataxonomic profiling was used to profile samples, targeting the V1 - V2 regions of 16S rRNA with samples run on a MiSeq platform. Reads were trimmed, combined and aligned to the Greengenes database. Microbial diversity and composition data were generated and analysed using Quantitative Insights Into Microbial Ecology (QIIME) and R software. 
40

41

42

43

44

45

46

47

48

49

50

51

52

53

54

55

56

57

58

59

60

61

62

63

64

65

66

67

68

69

70

71

72

73

74

75

76

77

78

79

Results. Child donor was the greatest predictor of microbiome variation between the stool samples, with all samples remaining identifiable to their child of origin despite the stool being stored under a variety of conditions. However, significant differences were observed in composition and diversity between preservation techniques, but intra-preservation technique variation was minimal for all preservation methods, and across the time-to-freezing range $(0-32$ h) used. Stool heterogeneity yielded no apparent microbiome differences.

Conclusions. Stool collected in a fieldwork setting for comparative microbiome analyses should ideally be stored as consistently as possible using the same preservation method throughout.

\section{Introduction}

Profiling faecal microbiota is now routinely applied to explore relationships between microbiota and host health status (Young, 2017). Since stool, including the microbiota, is subject to change post-collection, it is essential that samples are preserved in a way that minimizes microbial growth, degradation and contamination to ensure microbial associations being detected in comparative studies are not influenced by storage. The 'gold standard' for storing stool for microbiome analysis is cryopreserving at $-80^{\circ} \mathrm{C}$ without a buffer (Vandeputte et al. 2017). Preservation at $-20^{\circ} \mathrm{C}$ has also been proposed as appropriate (Song et al. 2016), although this may not be ideal for longer term storage (Bahl et al. 2012; Gorzelak et al. 2015). Whilst suitable for human studies in high income countries, cryopreservation is often not feasible for large scale projects in remote fieldwork settings, especially in low and middle-income countries (LMIC). Focusing on conditions more likely to be accessible in these settings, several studies have assessed the impact of storage under standard cold chain, i.e. $+4^{\circ} \mathrm{C}$ (Choo et al. 2015 ; Lauber et al. 2010; Penington et al. 2018; Tedjo et al. 2015), and 'room' (i.e. $25^{\circ} \mathrm{C}$ ) temperatures (Cardona et al. 2012; Guo et al. 2016; Lauber et al. 2010; Tal et al. 2017; Tedjo et al. 2015) prior to freezing. These approaches appear to be sufficient to maintain a representative metataxonomic 16S rRNA microbiota community profile in the short-term (up to 14 days post collection). However, to the best of our knowledge, there have been no studies determining the effect of real time temperature fluctuations commonly seen in tropical fieldwork environments. Since geographically separated populations have distinct microbiota compositions (Lee et al. 2014; Yatsunenko et al. 2012), it is reasonable to hypothesise that the microbiota in stool samples from different communities could also have different rates of abiotic change. Exploring the impact of time-to-freezing on gut microbiota profiles is therefore an important consideration for field studies in tropical LMIC, where the gut microbiome composition is less well established, temperature variation is more difficult to control, and collection standards are difficult to optimise.

Furthermore, access to laboratory consumables and resources are often limited, unreliable and potentially challenging to replenish in remote LMIC locations. Informed and realistic 
80 considerations must be made about the best practices for storage of stool samples in such

81 situations to maintain sample integrity. More recently, preservation solutions have been used in

82 an attempt to preserve DNA, and minimise microbial changes in stool after collection. Minimal

83 differences have been reported between different room-temperature storage preservation

84 solutions compared to immediately frozen raw stools (Blekhman et al. 2016; Dominianni et al.

85 2014; Wang et al. 2018). Another study reported that stool microbiome 16S rRNA profiles stored

86 in preservatives at ambient temperature for three days prior to freezing at $-80^{\circ} \mathrm{C}$ were

87 significantly different in composition and diversity compared to immediately frozen samples

88 without preservative (Choo et al. 2015). Although storage preservation was being compared, it is

89 possible that both time-to-freezing and abiotic factors influenced results. Understanding the

90 performance of preservation methods, as well as their impact in combination with time-to-

91 freezing, may be useful in settings susceptible to large temperature fluctuations, where cold

92 storage may be unreliable or unavailable.

93

Sample heterogeneity is another important consideration when trying to obtain representative microbiota profiles, as previous studies have indicated microbial profiles differ in different parts of the stool sample (Gorzelak et al. 2015; Wesolowska-Andersen et al. 2014). Therefore, ensuring samples collected are representative and consistent, particularly in fieldwork situations where homogenisation of the stool sample may be difficult due to limited resources, is another sampling consideration.

100

To address these crucial issues, we explored the influence of time-to-freezing, storage

preservation methodology, and stool heterogeneity on microbiome profiles for stool specimens

103

104

105

106

107

108

109

110

111

112

113

114

115

116

117

118

119

collected from three children within a Ugandan community representative of an LMIC fieldwork setting. Stool donor was found to be the greatest source of microbiota variation. Differences between the preservation method were also observed, but to a lesser extent.

\section{Materials \& Methods}

\section{Ethics Statement}

This study was approved by the University of Glasgow College of Medical Veterinary and Life Sciences Ethics Committee (project code 200160068), the Vector Control Division, Ministry of Health Uganda, Research Ethics Committee (reference: VCDREC/062) and the Uganda National Council for Science and Technology (UNCST-HS 2193). Informed signed or fingerprinted parental or guardian consent, and signed or fingerprinted assent from the study children was obtained prior to participation.

\section{Sample Collection}

One stool specimen was collected from three children, aged $12-14$, selected at random from Bugoto Lake View Primary School, Mayuge District, Uganda in March 2017. The sample from Child A was collected on day 1, and those from Child B and Child C on day 2. Outer surface, 
120 central inner and mixed stool samples ( $\sim 300 \mathrm{mg}$ each) were taken from each specimen and stored

121

122

123

124

125

126

127

128

129

130

131

132

133

134

135

136

137

138

139

140

141

142

143

144

145

146

147

148

149

150

151

152

153

154

155

156

157

158

159

separately in cryovials as raw stool (considered the standard), dispersed in absolute ethanol (approx. stool:ethanol ratio $=1: 6$ ) and dispersed in RNAlater (approx. stool:RNAlater ratio $=$ $1: 6)$, then frozen immediately on dry ice. Additionally coarsely homogenised stool from each donor were frozen on dry ice at 1.2, 4, 8, 16 and $32 \mathrm{~h}$ post collection for each storage preservation method (Table S1). taken from an individual stool specimen, had been processed into all the collection tubes for the relevant conditions to be tested, which was approximately $30 \mathrm{~min}$ after defecation. Prior to freezing on dry ice, stool was kept in cooler, shaded, well ventilated, indoor spaces as much as realistically possible. Within $48 \mathrm{~h}$ of freezing on dry ice, samples were transferred into a $-20^{\circ} \mathrm{C}$ freezer and later transported to the University of Glasgow on dry ice for further processing and analysis. Samples underwent one freeze-thaw cycle $(<30 \mathrm{~min})$ during weighing and, to the best of our knowledge, they remained frozen at $-20^{\circ} \mathrm{C}$ from collection until DNA was extracted approximately six months later. Cryovials used in the field containing only ethanol or RNAlater, without stool, were used as negative controls. Samples from two of the children were also stored using OMNIgene.GUT kits (DNA Genotek (Doukhanine et al. 2014)) as per manufacturer's instructions, and remained at ambient temperature until DNA was extracted approximately six (four in Uganda and two in the UK) months later ( three times the recommended 60 day stability recommendation for the kit (http://www/dnagenotek.com/us/products/collectionmicrobiome/omnigene-gut/OMR-200.html)). Details of samples and sample codes are shown in Table S1.

\section{Extraction of DNA from Stool}

The MPbio FastDNA ${ }^{\text {TM }}$ SPIN Kit for Soil (MPbio), was used to extract nucleic acids from 200 mg of stool with minor modifications to the method described by Alcon-Giner et al. 2017, as follows. An attempt was made to exclude large pieces of undigested vegetable matter from stool during the weighing process. Samples were homogenised using a TissueLyser II (Qiagen) at a speed setting of 25 , and a 2 min centrifugation was used after addition of binding matrix. DNA concentration was quantified using a NanoDrop 1000 fluorimeter (Thermo Fisher Scientific).

\section{S Library Preparation}

A modification of the Illumina $16 \mathrm{~S}$ metagenomic sequencing library preparation protocol (https://support.illumina.com/documents/documentation/chemistry_documentation/16s/16smetagenomic-library-prep-guide-15044223-b.pdf) was used to prepare the DNA library. PCR was used to amplify the V1 - V2 regions of the 16S rRNA gene, chosen because they were better at detecting bacterial species of interest from stool for future studies (eg. Bifidobacterium (Alcon-Giner et al. 2017)). The primers used were: 16SV1 forward primer (5'TCGTCGGCAGCGTCAGATGTGTATAAGAGACAGAGMGTTYGATYMTGGCTCAG -3') and $16 \mathrm{SV} 2$ reverse primer $\left(5^{\prime}\right.$ GTCTCGTGGGCTCGGAGATGTGTATAAGAGACAGCTGCCTCCCGTAGGAGT -3’).

Peer] reviewing PDF | (2019:05:37936:1:1:NEW 3 Oct 2019) 
160 Each reaction was performed in a final volume of $25 \mu \mathrm{L}$ consisting of 1x KAPA HiFi HotStart

161

162

163

164

165

166

167

168

169

170

171

172

173

174

175

176

177

178

179

180

181

182

183

184

185

186

187

188

189

190

191

192

193

194

195

196

197

198

199

ReadyMix (KAPA Biosystems), $0.5 \mu \mathrm{M}$ of each primer and $12.5 \mathrm{ng}$ of sample DNA.

Thermocycler conditions were used as follows: $95^{\circ} \mathrm{C}$ for $5 \mathrm{~min}$, followed by 26 cycles of $95^{\circ} \mathrm{C}$ for $30 \mathrm{~s}$ and $60^{\circ} \mathrm{C}$ for $1 \mathrm{~min}$. Samples were then held at $10^{\circ} \mathrm{C}$ in the PCR machine, before being stored at $4^{\circ} \mathrm{C} . \mathrm{H}_{2} \mathrm{O}$ sample controls were included as negative controls during the first round of PCR to monitor non-specific amplification.

Each PCR product was purified by mixing with a $0.90 x$ PCR product volume of High Prep PCR beads (MAGBIO). After a $10 \mathrm{~min}$ incubation, sample tubes were placed on a magnetic stand and left until the supernatant became clear. The supernatant was then removed and the beads were washed twice with freshly prepared $80 \%$ ethanol, and then left to dry for 15 min to allow residual ethanol to evaporate. The sample tubes were removed from the stand and the beads were then resuspended in $20 \mu \mathrm{L}$ Tris buffer $\mathrm{pH} 8.5$, and incubated for 2 min before being placed back on the magnetic stand. Once clear, the supernatant was transferred to a fresh tube and the DNA concentration quantified using the Quant-iT PicoGreen dsDNA Assay (https://assets.thermofisher.com/TFS-Assets/LSG/manuals/mp07581.pdf) (Thermo Fisher Scientific).

A second PCR step was then used to barcode each sample. PCR reactions were performed in a final volume of $50 \mu \mathrm{L}$ consisting of 1x KAPA HiFi HotStart ReadyMix, $5 \mu \mathrm{L}$ of each of two Nextera XT Index Kit Set A (Illumina) indices, with each sample having a unique combination, and $10 \mathrm{ng}$ of post-PCR1 sample DNA. Thermocycler conditions used were as follows: $95^{\circ} \mathrm{C}$ for $3 \mathrm{~min}$; followed by 8 cycles of $95^{\circ} \mathrm{C}, 55^{\circ} \mathrm{C}$ and $72^{\circ} \mathrm{C}$ for $30 \mathrm{~s}$ each; with a final step of $72^{\circ} \mathrm{C}$ for $5 \mathrm{~min}$. Samples were then held at $10^{\circ} \mathrm{C}$ in the PCR machine, before being stored at $4^{\circ} \mathrm{C}$.

Samples were cleaned with High Prep PCR beads as described above and then combined to form an equimolar sample library. The Wizard SV Gel and PCR Clean-Up System Kit (Promega) was used to purify the DNA library prior to sequencing, as per manufacturer's instructions (https://www.promega.co.uk/-/media/files/resources/protocols/technical-bulletins/101/wizard-svgel-and-pcr-clean-up-system-protocol.pdf?la=en) using a band size of $\sim 435 \mathrm{bp}$. DNA concentration was then measured using a Bioanalyser 2100 (Agilent).

\section{Sample Sequencing and Analysis}

Samples were sequenced using the Illumina MiSeq platform (Glasgow Polyomics) with two $\mathrm{x}$ 300 bp paired-end read lengths with up to 100,000 reads per sample (MiSeq V3 600 cycle kit (Illumina)). Using cutadapt software (Martin 2011) in Python version 2.7, barcode sequences were removed, reads trimmed to a minimum quality score of 20, and then reads less than $250 \mathrm{bp}$ in length were discarded (Code $S 1$ ). Forward and reverse reads were combined using PANDAseq (Masella et al. 2012) for each sample before all files were merged into one file containing all samples (Code S1). Quantitative Insights Into Microbial Ecology (QIIME) software version 1.9.1

Peer) reviewing PDF | (2019:05:37936:1:1:NEW 3 Oct 2019) 
200 (Caporaso et al. 2010) in Python version 2.7 was used to analyse the data. Operational

201 Taxonomic Units were assigned with 97\% clustering to the Greengenes database version 13.8

202 (DeSantis et al. 2006) for 16S rRNA gene alignment. Sequences aligning to mitochondria or

203 chloroplast sequences were screened for and removed from the dataset. Custom scripts in QIIME

204 were used to analyse relative taxonomic abundance, and alpha and beta diversity measures (Code

$205 S 1)$ at a sequencing depth of 10,000 reads per sample. Pairwise comparisons of beta diversity

206 measures (weighted (Lozupone et al. 2007) and unweighted (Lozupone et al. 2005) UniFrac)

207 were made using 999 Monte Carlo permutations (MCP). The Linear Discriminant Effect Size

208 (LEfSe) (Segata et al. 2011) algorithm was performed to identify taxonomic groups associated

209 with the variables measured ( $p<0.01$, Linear Discriminant Analysis (LDA) score $(\log 10 \geq 2)$ ).

210 To be included in the results, each variable must have met the inclusion criteria $(p<0.01$, LDA

211 score $(\log 10) \geq 2)$ within each child, as well as when averaged across all three children. Higher

212 taxonomic levels were excluded where it was assumed that a lower taxonomic level was

213 accountable for the observed change. These situations were where a higher taxonomic level had

214 a less significant or equal change in relative abundance compared to a lower taxonomic level

215 classified to the higher taxonomy by LEfSe analysis. However, if the higher taxonomic level had

216 a more significant $\mathrm{p}$ value it was retained. Kruskal-Wallis tests to compare read counts

217 (significantly different if $\mathrm{p}<0.05$ ) were performed in $\mathrm{R}$ version 3.4.2 (R Core Team 2017) and

218 graphs were generated using the ggplot2 package (Wickham et al. 2018). All data are provided

219 as supplemental information.

220

221 Alpha diversity scores (species richness, Shannon and Simpson), generated using standard

222 parameters in QIIME 1.9.1, were analysed by generating linear mixed effect models using the

223 lme4 package (Bates et al. 2018) in R version 3.4.2 (R Core Team 2017) to identify important

224 predictors of alpha diversity. The lmerTest package (Kuznetsova et al. 2017) was used to

225 determine the significance of these model components. Two maximal models were constructed

226 and included all the fixed effects (preservation method, time-to-freezing and stool region) and

227 their interactions with child replicate as a random effect, the first included time as a continuous

228 variable and the second included time as a factor. Backward elimination was used for sequential

229 removal of non-significant variables, to obtain the minimal statistically significant model

230 (Burnham et al. 2011) (Code S2).

231

232 Taxonomic abundance graphs and LEfSe plots generated with QIIME were recreated in R using

233 the ggplot2 package (Wickham et al. 2018). Principal Coordinates Analysis (PCoA) plots were

234 generated using Emperor Software (Vázquez-Baeza et al. 2013) within QIIME.

235

236

237

Due to the low number $(\mathrm{n}=2)$ of OMNIgene.GUT samples taken, and because OMNIgene.GUT

238 samples were only taken from two out of the three children, these samples were excluded from the above analyses and analysed separately. 


\section{Results}

\section{Samples processing and microbiome sequencing}

242 In total 87 stool samples were collected for analysis and librar prepared. After sample

243 exclusion, trimming and alignment (Fig. S1, Fig. S2 and Table s/) there was an average of

24467,575 (range 19,083 - 466,807) reads per sample $(\mathrm{n}=85)$.

245

246

247

\section{Microbiome profiles vary between individual children}

248

249

250

251

252

253

254

255

256

257

258

259

260

261

262

263

264

265

266

267

268

269

270

271

272

273

274

275

276

277

278

279

Each child had a distinct microbiome signature (Fig. $1 A$ and Fig. $1 B$ ) that was apparent at all taxonomic levels, from phylum (Fig. $2 A$ and Table S2) to genus (Fig. $2 B$ and Data S1) level regardless of the preservation method used and the time-to-freezing duration. The most abundant phyla were Bacteroidetes in child A (40.7\%) and child B (36.9\%), and Firmicutes in child C (34.1\%); followed by Firmicutes in child A (40.1\%), Proteobacteria in child B (30.2\%) and Bacteroidetes in child C (28.7\%). LEfSe identified several bacterial taxa significantly associated with each individual child (Data $S 2$ ). PCoA analysis using qualitative (presence/absence) differences (unweighted UniFrac, Fig. 1A) confirmed that the clustering of bacterial sequences within individual children was significantly different (MCP for all child comparisons $\mathrm{p}=\leq$ 0.001) (Fig. 1A). Children were also significantly different by relative abundance weighted UniFrac (MCP for all child comparisons $\mathrm{p}=\leq 0.001)($ Fig. 1B).

\section{Microbiome profiles vary by stool storage method used}

The samples stored as raw stool had a mean average of 80,822 reads per sample (range 26,270466,$807 ; 35$ samples), samples in ethanol had a mean average of 62,983 reads per sample (range 24,215 - 140,356; 24 samples), and samples in RNAlater had a mean average of 53,981 reads per sample (range 19,083 - 100,934; 26 samples) (Fig. S3). The number of read counts was not significantly different between preservation methods using a Kruskal-Wallis test.

The within-individual variation between samples stored under different preservation methods was less than that observed between individuals (Fig. 1A, Fig 1B and Fig. 2). Intra-storage preservation method microbiota abundance compositions were similar over the time points examined $(0-32 \mathrm{~h}$ ) for each of the preservation methods used (Fig. 2), suggesting relative stability. There were twelve taxonomic groups significantly associated with raw stool (all $\mathrm{p}<$ 0.01 , LDA score ( $\log 10 \geq 2$ ), Data S3). Eight and eleven taxonomic groups were positively associated with ethanol and RNAlater storage respectively, compared to raw stool alone (all $p<$ 0.01 , LDA score $(\log 10 \geq 2)$ (Fig. 3 and Data S3). Seven of the taxonomic groups that were significantly elevated in relative abundance were shared in the samples stored in ethanol and RNAlater (Fig. 3 and Data S3).

Microbiome diversity under different preservation methods were found to differ significantly within each child by qualitative unweighted UniFrac analysis (MCP; all comparisons $p \leq 0.001$ ). The unweighted Unifrac distances within ethanol samples were found to be significantly 
280 different compared to within RNAlater samples (child A: $p=0.003$, child $\mathrm{B}: \mathrm{p}=0.041$, child C: $281 \mathrm{p}=0.035$ ) or within raw stool samples (child A: $\mathrm{p}=0.02$, child $\mathrm{B}: \mathrm{p}=0.038$, child $\mathrm{C}: \mathrm{p}=\leq$ 282 0.001). UniFrac metrics within raw stool samples were not significantly different to metrics 283 within RNAlater samples apart from in child C $(\mathrm{p}=\leq 0.001)$. MCP analysis of unweighted

284

285

286

287

288

289

290

291

292

293

294

295

296

297

298

299

300

301

302

303

304

305

306

307

308

309

310

311

312

313

314

315

316

317

318

UniFrac comparisons across all three children also revealed significant differences between raw stool and RNAlater storage methods (raw stool vs. raw stool:raw stool vs. RNAlater, $\mathrm{p}=0.005$; RNAlater vs. RNAlater:RNAlater vs. raw stool, $\mathrm{p}=0.01$ ), and raw stool and ethanol storage (raw stool vs. raw stool: raw stool vs. ethanol, $\mathrm{p}=0.026)($ Fig. $1 C$ ) despite distinct separation by child (Fig. 1A).

When UniFrac measures were weighted by relative sequence abundance within each child and as an average of all three children, all preservation method comparisons by MCP were found to be significantly distant from each other (for all comparisons $\mathrm{p} \leq 0.001$ ) (Fig. 1B and Fig. 1D). Separation within raw stool samples was also found to be significantly different compared to separation within ethanol storage (MCP; child A: $p=0.017$, child B: $p=0.041$, child $\mathrm{C}: \mathrm{p}=\leq$ 0.001 , all children: $p=0.005$ ). In child $C$ within RNAlater metrics were found to be significantly different by MCP compared to within raw stool metrics $(\mathrm{p}=\leq 0.001)$, however, this was not observed in child A or child B. No significant differences were observed by MCP between within RNAlater and within ethanol weighted UniFrac metrics.

\section{Microbiome profiles remain relatively stable over time}

Relative bacterial abundance and composition remained relatively stable over time-to-freezing across all storage techniques by LEfSe analysis when time-to-freezing was included as a continuous variable (Fig. 4). However, LEfSe analysis indicated a significantly increased relative abundance of two bacterial genera in raw stool samples at $32 \mathrm{~h}$ time-to-freezing when time-tofreezing was included as a categorical variable: Sediminibacterium $(\mathrm{p}=0.0016, \mathrm{LDA}(\log 10)=$ 2.35) (Fig. 5A) and Rummeliibacillus $(\mathrm{p}=0.0012$, LDA $(\log 10)=2.35)$ (Fig. 5B). No significant categorical time-to-freezing effects were identified in ethanol or RNAlater samples by LEfSe analysis. No apparent time clustering was observed by PCoA using UniFrac metrics. No significant differences by weighted or unweighted UniFrac metrics using MCP were observed when comparing $0 \mathrm{~h}$ sample metrics to any other time-to-freezing time point or vice versa.

\section{No significant differences in microbiome composition were observed between inner, outer and mixed regions of stool samples}

Microbiome profiles of the mixed stool samples were similar to inner and outer stool samples, from phylum through to genus level (Fig. 6). Significant differences between stool regions were not observed in this study by LEfSe regardless of whether the data were analysed by child, storage preservation method or as a whole. No significant associations were generated when LEfSe analysis was performed and no apparent clustering was identified by PCoA analysis of 
319 UniFrac diversity metrics. Weighted and unweighted UniFrac comparisons showed no

320 significant differences using MCP.

321

322

Modelling indicates storage method influences stool alpha diversity

323

Linear mixed effect models were constructed to detect variables associated with alpha diversity

324 metrics (Code S2, Table S3). None of the final models identified stool region or time (included as

325 a fixed or a continuous variable) to be significant predictors of alpha diversity. Individual

326 children included as a random effect alone was the only variable shown to influence Shannon

327 diversity and Simpson diversity.

328

329

330

331

332

$$
\begin{aligned}
& \text { Shannon } \sim 1+(1 \mid \text { child }) \\
& \text { Simpson } \sim 1+(1 \mid \text { child })
\end{aligned}
$$

333

334

335

336

337

338

339

340

341

342

343

344

345

346

347

348

349

350

351

352

353

354

355

356

357

358

Preservation method was identified to be a significantly important model component for the model predicting species richness $(\mathrm{p}=2.871 \mathrm{e}-13)$ with stool stored in RNAlater having the highest average richness, followed by raw stool and stool stored in ethanol (Table S3). Compared to the null species richness model, $50.2 \%$ of species richness variation was accounted for by the final species richness model.

\section{Species Richness $\sim 1+$ preservation method $+(1 \mid$ child $)$}

\section{OMNIgene.GUT sample performance}

Samples stored using OMNIgene.GUT kits had a mean average of 77,089 reads per sample (child $\mathrm{B}=102,227$; child $\mathrm{C}=51,951$ ). The samples clustered by PCoA analysis to the relevant children from which they were taken (Fig. S2). Relative abundance profiles were also representative of the microbiome profiles from each child (Fig. S4).

\section{Discussion}

As has been previously observed in stool microbiome studies (Blekhman et al. 2016; Carroll et al. 2012; Dominianni et al. 2014; Guo et al. 2016; Lauber et al. 2010; Penington et al. 2018; Wang et al. 2018), the sample donor was found to be the greatest predictor of gut microbiome variation amongst the variables studied here. Samples were identifiable to each child regardless of storage method, time-to-freezing or stool region (Fig.1 A, Fig. 1B and Fig. 2) (Blekhman et al. 2016; Carroll et al. 2012; Dominianni et al. 2014; Guo et al. 2016; Lauber et al. 2010; Penington et al. 2018; Wang et al. 2018). Individual child (included as a random effect) was also the only predictor of Shannon diversity and Simpson diversity from the variables measured. Multiple factors, including diet (David et al. 2014) and demographics (Yatsunenko et al. 2012), not recorded in this study, have been shown to influence microbial status within an individual, and each individual will have a unique combination of contributing factors. Individuality is therefore an important consideration when planning comparative microbial studies (i.e. between healthy 
359

360

361

362

363

364

365

366

367

368

369

370

371

372

373

374

375

376

377

378

379

380

381

382

383

384

385

386

387

388

389

390

391

392

393

394

395

396

397

398

and diseased states) to ensure enough participants are recruited into studies so that the obtained data are informative about the question of interest.

Although the microbiome diversity under different preservation methods clustered by child, both weighted and unweighted UniFrac metrics also indicated that samples stored by different preservation methods were significantly distant within each of the children (Fig. 1A and Fig. 1B). PCoA of UniFrac metrics further revealed clustering by preservation method despite child variation (Fig. 1C and Fig. 1D), suggesting each preservation method acts similarly across each child. Despite significant differences between within-preservation-method UniFrac metrics, there was no trend in preservation method performance across each of the children. This could be due to unique microbial profiles of the children being suited to different types of storage or attributed to the randomness between samples taken within a specimen, which may also account for the significant differences in UniFrac metrics observed in child C. Modelling also identified that preservation method had a significant effect on species richness. Significant differences in the microbial profiles from stool stored in RNAlater and ethanol were identified when compared to samples stored raw, that were considered 'gold standard' for this study (Fig. 3). These differences appeared to remain relatively stable across time-to-freezing (Fig. 4) and were evident even at time zero, when samples were first frozen by 30 min post-defecation, suggesting that changes occurred rapidly, within a few minutes, after the addition of stool to preservative. All of the bacterial levels correlated to ethanol and/or RNAlater preservation identified by LEfSe, of which the two methods shared seven groups of the eight and eleven groups respectively (Fig. 3), were associated with some form of anaerobic metabolism. Anaerobic bacteria are possibly overrepresented or better preserved than aerobic species in stool stored using these methods.

Preservative exposure therefore may influence microbial profiles obtained from stool via a common physical mechanism, which favours the preservation of some bacterial taxa over others, making stool stored in various preservatives more similar in microbial structure and comparable to each other. This is in agreement with a study that found samples stored in preservatives were more likely to cluster together by PCoA, based on Bray-Curtis similarity distances (Choo et al. 2015). Alternatively, it is possible that PCR product amplification of certain species was altered by residual ethanol or RNAlater salts despite care being taken to limit these PCR contaminants during the DNA extraction process. RNAlater has been reported to reduce DNA yield by qPCR (Gorzelak et al. 2015) and 16S rRNA DNA amplification purity (Dominianni et al. 2014) in microbiota studies. However, microbiota variation between samples within preservation method groups was low (Fig. 1, Fig. 2 and Fig. 4) and DNA concentrations were standardized across the samples in our study, suggesting that at least some of the associations observed are due to the stool preservation method.

Irrespective of preservation techniques, microbiome profiles remained adequately stable for up to $32 \mathrm{~h}$ in tropical ambient temperatures when compared to their baseline $(0 \mathrm{~h}$, frozen by $30 \mathrm{~min}$ post-defecation), with only two minor, albeit significant, changes in relative abundance arising 
399

400

401

402

403

404

405

406

407

408

409

410

411

412

413

414

415

416

417

418

419

420

421

422

423

424

425

426

427

428

429

430

431

432

433

434

435

436

437

438

by $32 \mathrm{~h}$ in raw stool samples when time-to-freezing was included as a categorical variable (Fig. 5). The identified increase in one of these, Rummeliibacillus however, is likely strongly influenced by one sample, CM32A (Fig. 5B and Table S1), making further studies necessary to determine the reproducibility and impact of this finding. Sampling at more regular intervals between $16-32 \mathrm{~h}$ would reveal if these increases are continuous over time and when they might start to occur.

Models did not identify time to be a significant predictor as a continuous or factorial component for species richness, Shannon diversity or Simpson diversity. These findings are in agreement with Tedjo et al. (2015) and Tal et al. (2017) who found no significant differences in diversity measure scores after $24 \mathrm{~h}$ (Shannon and Chao1) and $96 \mathrm{~h}$ (Shannon, Simpson and Chao1) of room temperature storage respectively. Storage at room temperature did significantly reduce weighted Shannon and Weaver diversity scores by $17 \%$ after $8 \mathrm{~h}$ at room temperature in another study (Ott et al. 2004). Diversity scores however, should always be considered in the context of specific bacteria profiles since the presence and absence of bacteria could change over time but the derived diversity score could remain stable.

No significant observations in microbial profiles were identified between the stool regions, a finding in contrast to previous studies reporting differences using qPCR (Gorzelak et al. 2015) and associations between microbial richness and stool consistency (Vandeputte et al. 2016). The Bristol stool chart (O'Donnell et al. 1990) defines seven levels of stool consistency and water content from type 1 (solid lumps) to a type 7 (watery liquid). It is plausible that inner and outer stool regions at the higher end of the scale are likely to be more uniform than stool at the lower end of the scale, and more difficult to define inner and outer stool regions at higher stool values. Stool samples collected from the Ugandan children for this study, although not formally graded, commonly fell into the higher end of the Bristol stool chart guide. Classifying stool specimens prior to sectioning may be a useful factor to explore in future work, along with other associations such as diet or health status. Stool size may also impact heterogeneity with the inner and outer regions of 'larger' stools being more distinguishable. This may explain why Gorzelak et al. (2015) and Vandeputte et al. (2016) obtained significant differences as their samples were collected from adults, who presumably produced larger stool specimens at the lower end of the Bristol stool chart than the LMIC child samples collected in this study. Whilst we did not see any differences associated with stool region, suggesting crude mixing is sufficient to maintain a representative microbiome in situations where specialized equipment is unavailable, the number of specimens collected in this study was small $(n=3)$. Therefore, there may not have been enough replicates to detect changes in stool heterogeneity in this study, and more samples ranging in different sizes may need to be studied to fully understand the impact of stool heterogeneity.

\section{Conclusions}


439 Stool samples collected for microbiome analyses are subject to biological change upon exposure

440 to abiotic differences in the environment. This study examined the impact of different stool

441 storage conditions on the human gut microbiome composition in a tropical LMIC, resource-

442 limited setting. Stool donor accounted for the greatest amount of variation seen in the gut

443 microbiota. Stool storage preservation method significantly influenced the bacterial profiles

444 obtained, however, all samples remained identifiable to their child of origin. Stool stored at

445 ambient temperature for up to $32 \mathrm{~h}$ did not significantly influence diversity and had minimal

446 changes upon microbiota composition, which remained relatively stable across time-to-freezing

447 regardless of preservation method used. No apparent differences were observed between outer,

448 inner or mixed stool regions taken however, sample size was small. Overall, comparative studies

449 involving stool storage for microbiome analysis should be performed as consistently as possible

450 in the tropical resource-limited settings, using the same preservation method throughout.

451

452

453

454

455

456

457

458

459

460

461

462

463

464

465

466

467

468

469

470

471

472

473

474

475

476

477

478

479

480

481

\section{Acknowledgements}

We would like to thank our Vector Control Division fieldwork driver Lugigana 'Fiddi' Andrew for his dedication and enthusiasm. Thank you to the community of Bugoto, Uganda, for making us feel welcome, and to the children for participating in our study. Thank you to Dr. David McGuinness at Glasgow Polyomics for providing bioinformatics training and technical support.

The authors Lindsay J Hall, Lisa C Ranford-Cartwright and Poppy H L Lamberton contributed significantly and equally to the design of the study as well as to the analysis and interpretation of the data and drafting of the manuscript, but in different ways based on their different expertise.

\section{References}

Alcon-Giner C, Caim S, Mitra S, Ketskemety J, Wegmann U, Wain J, Belteki G, Clarke P, and Hall LJ. 2017. Optimisation of 16S rRNA gut microbiota profiling of extremely low birth weight infants. BMC Genomics 18:841. 10.1186/s12864-017-4229-x

Bahl MI, Bergström A, and Licht TR. 2012. Freezing fecal samples prior to DNA extraction affects the Firmicutes to Bacteroidetes ratio determined by downstream quantitative PCR analysis. FEMS Microbiol Lett 329:193-197. 10.1111/j.1574-6968.2012.02523.x

Bates D, Maechler M, Bolker B, Walker S, Christensen, B. RH, Singmann H, Dai B, Scheipl F, Grothendieck G, Green P, and Fox J. 2018. lme4: Linear Mixed-Effects Models using 'Eigen' and S4.R package version 1.1 - 17. URL: https://CRAN.R-project.org/package=lme14.

Blekhman R, Tang K, Archie EA, Barreiro LB, Johnson ZP, Wilson ME, Kohn J, Yuan ML, Gesquiere L, Grieneisen LE, and Tung J. 2016. Common methods for fecal sample storage in field studies yield consistent signatures of individual identity in microbiome sequencing data. Sci Rep 6:31519. 10.1038/srep31519

Burnham KP, Anderson DR, and Huyvaert KP. 2011. AIC model selection and multimodel inference in behavioural ecology: some background, observations, and comparisons. Behavioural Ecology and Sociobiology 65:23-35. 10.1007/s00265-010-1029-6

Caporaso JG, Kuczynski J, Stombaugh J, Bittinger K, Bushman FD, Costello EK, Fierer N, Peña AG, Goodrich JK, Gordon JI, Huttley GA, Kelley ST, Knights D, Koenig JE, Ley RE, Lozupone CA, McDonald D, Muegge BD, Pirrung M, Reeder J, Sevinsky JR, Turnbaugh PJ, Walters WA, 
482

483

484

485

486

487

488

489

490

491

492

493

494

495

496

497

498

499

500

501

502

503

504

505

506

507

508

509

510

511

512

513

514

515

516

517

518

519

520

521

522

523

524

525

526

527

528

529

530

531

532
Widmann J, Yatsunenko T, Zaneveld J, and Knight R. 2010. QIIME allows analysis of highthroughput community sequencing data. Nat Methods 7:335-336. 10.1038/nmeth.f.303

Cardona S, Eck A, Cassellas M, Gallart M, Alastrue C, Dore J, Azpiroz F, Roca J, Guarner F, and Manichanh C. 2012. Storage conditions of intestinal microbiota matter in metagenomic analysis. BMC Microbiol 12:158. 10.1186/1471-2180-12-158

Carroll IM, Ringel-Kulka T, Siddle JP, Klaenhammer TR, and Ringel Y. 2012. Characterization of the fecal microbiota using high-throughput sequencing reveals a stable microbial community during storage. PLoS One 7:e46953. 10.1371/journal.pone.0046953

Choo JM, Leong LE, and Rogers GB. 2015. Sample storage conditions significantly influence faecal microbiome profiles. Sci Rep 5:16350. 10.1038/srep16350

David LA, Maurice CF, Carmody RN, Gootenberg DB, Button JE, Wolfe BE, Ling AV, Devlin AS, Varma Y, Fischbach MA, Biddinger SB, Dutton RJ, and Turnbaugh PJ. 2014. Diet rapidly and reproducibly alters the human gut microbiome. Nature 505:559-563. 10.1038/nature12820

DeSantis TZ, Hugenholtz P, Larsen N, Rojas M, Brodie EL, Keller K, Huber T, Dalevi D, Hu P, and Andersen GL. 2006. Greengenes, a chimera-checked 16S rRNA gene database and workbench compatible with ARB. Appl Environ Microbiol 72:5069-5072. 10.1128/AEM.03006-05

Dominianni C, Wu J, Hayes RB, and Ahn J. 2014. Comparison of methods for fecal microbiome biospecimen collection. BMC Microbiol 14:103. 10.1186/1471-2180-14-103

Doukhanine EB, A, Merino C, and Pozza L. 2014. OMNIgene ${ }^{\circledR} \cdot$ GUT enables reliable collection of high quality fecal samples for gut microbiome studies. 2015-16:URL: https://www.dnagenotek.com/us/pdf/PD-WP-00040.pdf.

Gorzelak MA, Gill SK, Tasnim N, Ahmadi-Vand Z, Jay M, and Gibson DL. 2015. Methods for improving human gut microbiome data by reducing variability through sample processing and storage of stool. PLoS One 10:e0134802. 10.1371/journal.pone.0134802

Guo Y, Li SH, Kuang YS, He JR, Lu JH, Luo BJ, Jiang FJ, Liu YZ, Papasian CJ, Xia HM, Deng HW, and Qiu X. 2016. Effect of short-term room temperature storage on the microbial community in infant fecal samples. Sci Rep 6:26648. 10.1038/srep26648

Kuznetsova A, Brockho PB, and Christensen RHB. 2017. lmerTest Package: Tests in Linear Mi: Effects Models. Journal of Statistical Software 82. 10.18637/jss.v082.i13

Lauber CL, Zhou N, Gordon JI, Knight R, and Fierer N. 2010. Effect of storage conditions on the assessment of bacterial community structure in soil and human-associated samples. FEMS Microbiol Lett 307:80-86. 10.1111/j.1574-6968.2010.01965.x

Lee SC, Tang MS, Lim YA, Choy SH, Kurtz ZD, Cox LM, Gundra UM, Cho I, Bonneau R, Blaser MJ, Chua KH, and Loke P. 2014. Helminth colonization is associated with increased diversity of the gut microbiota. PLoS Negl Trop Dis 8:e2880. 10.1371/journal.pntd.0002880

Lozupone CA, Hamady M, Kelley, ST, and Knight R. 2007. Quantitative and qualitative beta diversity measures lead to different insights into factors that structure microbial communities. Appl Environ Microbiol 73: 1576-1585. 10.1128/AEM.01996-06

Lozupone CA, and Knight R. 2005. UniFrac: a new phylogenetic method for comparing microbial communities. Appl Environ Microbiol 71: 8228-8235. 10.1128/AEM.71.12.8228-8235.2005

Martin M. 2011. Cutadapt removes adapter sequences from high-throughput sequencing reads. EMBnetjournal 17:10-12. https://doi.org/10.14806/ej.17.1.200

Masella AP, Bartram AK, Truszkowski JM, Brown DG, and Neufeld JD. 2012. PANDAseq: paired-end assembler for illumina sequences. BMC Bioinformatics 13:31. 10.1186/1471-2105-13-31

O'Donnell LJ, Virjee J, and Heaton KW. 1990. Detection of pseudodiarrhoea by simple clinical assessment of intestinal transit rate. BMJ 300:439-440.

Ott SJ, Musfeldt M, Timmis KN, Hampe J, Wenderoth DF, and Schreiber S. 2004. In vitro alterations of intestinal bacterial microbiota in fecal samples during storage. Diagn Microbiol Infect Dis 50:237-245. 10.1016/j.diagmicrobio.2004.08.012

Penington JS, Penno MAS, Ngui KM, Ajami NJ, Roth-Schulze AJ, Wilcox SA, Bandala-Sanchez E, Wentworth JM, Barry SC, Brown CY, Couper JJ, Petrosino JF, Papenfuss AT, Harrison LC, and

Peer] reviewing PDF | (2019:05:37936:1:1:NEW 3 Oct 2019) 
533

534

535

536

537

538

539

540

541

542

543

544

545

546

547

548

549

550

551

552

553

554

555

556

557

558

559

560

561

562

563

564

565

566

567

568

569
Group* ES. 2018. Influence of fecal collection conditions and 16S rRNA gene sequencing at two centers on human gut microbiota analysis. Sci Rep 8:4386. 10.1038/s41598-018-22491-7

$\mathrm{R}$ Core Team. 2017. R: a language and environment for statistical computing: version $3.4 .2 \mathrm{R}$ foundation for statistical computing: Vienna, Austria.:URL: https://www.R-project.org/.

Segata N, Izard J, Waldron L, Gevers D, Miropolsky L, Garrett WS, and Huttenhower C. 2011. Metagenomic biomarker discovery and explanation. Genome Biol 12:R60. 10.1186/gb-2011-126-r60

Song SJ, Amir A, Metcalf JL, Amato KR, Xu ZZ, Humphrey G, and Knight R. 2016. Preservation methods differ in fecal microbiome stability, affecting suitability for field studies. mSystems 1. 10.1128/mSystems.00021-16

Tal M, Verbrugghe A, Gomez DE, Chau C, and Weese JS. 2017. The effect of storage at ambient temperature on the feline fecal microbiota. BMC Vet Res 13:256. 10.1186/s12917-017-1188-z

Tedjo DI, Jonkers DM, Savelkoul PH, Masclee AA, van Best N, Pierik MJ, and Penders J. 2015. The effect of sampling and storage on the fecal microbiota composition in healthy and diseased subjects. PLoS One 10:e0126685. 10.1371/journal.pone.0126685

Vandeputte D, Falony G, Vieira-Silva S, Tito RY, Joossens M, and Raes J. 2016. Stool consistency is strongly associated with gut microbiota richness and composition, enterotypes and bacterial growth rates. Gut 65:57-62. 10.1136/gutjnl-2015-309618

Vandeputte D, Tito RY, Vanleeuwen R, Falony G, and Raes J. 2017. Practical considerations for largescale gut microbiome studies. FEMS Microbiol Rev 41:S154-S167. 10.1093/femsre/fux027

Vázquez-Baeza Y, Pirrung M, Gonzalez A, and Knight R. 2013. EMPeror: a tool for visualizing highthroughput microbial community data. Gigascience 2:16. 10.1186/2047-217X-2-16

Wang Z, Zolnik CP, Qiu Y, Usyk M, Wang T, Strickler HD, Isasi CR, Kaplan RC, Kurland IJ, Qi Q, and Burk RD. 2018. Comparison of fecal collection methods for microbiome and metabolomics studies. Front Cell Infect Microbiol 8:301. 10.3389/fcimb.2018.00301

Wesolowska-Andersen A, Bahl MI, Carvalho V, Kristiansen K, Sicheritz-Pontén T, Gupta R, and Licht TR. 2014. Choice of bacterial DNA extraction method from fecal material influences community structure as evaluated by metagenomic analysis. Microbiome 2:19. 10.1186/2049-2618-2-19

Wickham H, Chang W, Henry L, Pedersen TL, Takahashi K, Wilke C, Woo K, and and R Studio. 2018. ggplot2: create elegant data visualisations using the grammar of graphics.R Package version 3. 1. 0. URL: https://CRAN.R-project.org/package=ggplot2.

Yatsunenko T, Rey FE, Manary MJ, Trehan I, Dominguez-Bello MG, Contreras M, Magris M, Hidalgo G, Baldassano RN, Anokhin AP, Heath AC, Warner B, Reeder J, Kuczynski J, Caporaso JG, Lozupone CA, Lauber C, Clemente JC, Knights D, Knight R, and Gordon JI. 2012. Human gut microbiome viewed across age and geography. Nature 486:222-227. 10.1038/nature11053 
Figure 1

Samples cluster by individual and storage method using principal coordinate (PC) analysis of unweighted ( $A$ and $C$ ) and weighted ( $B$ and $D)$ unifrac measures.

Ellipses enclose samples from the same individual $(A$ and $B)$. Storage method: Red $=$ raw stool, Blue $=$ ethanol and Orange $=$ RNAlater. $\mathrm{PC} 1, \mathrm{PC} 2$ and $\mathrm{PC} 3(\mathrm{~A}$ and $\mathrm{B}$ ); PC2, PC3 and PC4 (C); and PC1, PC2 and PC4 (D).

A

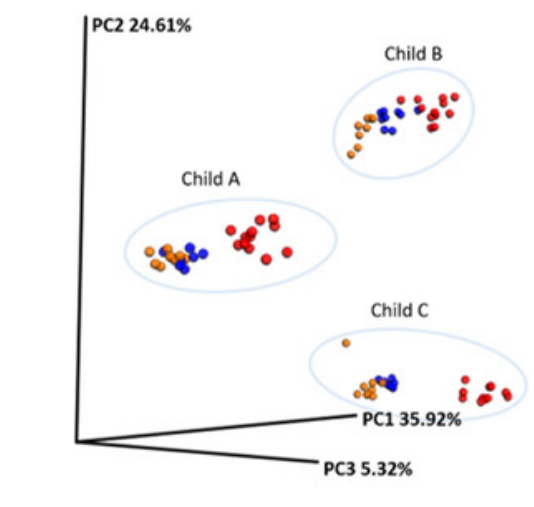

C

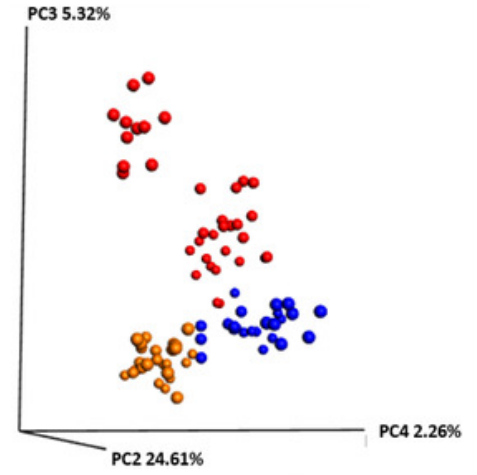

B
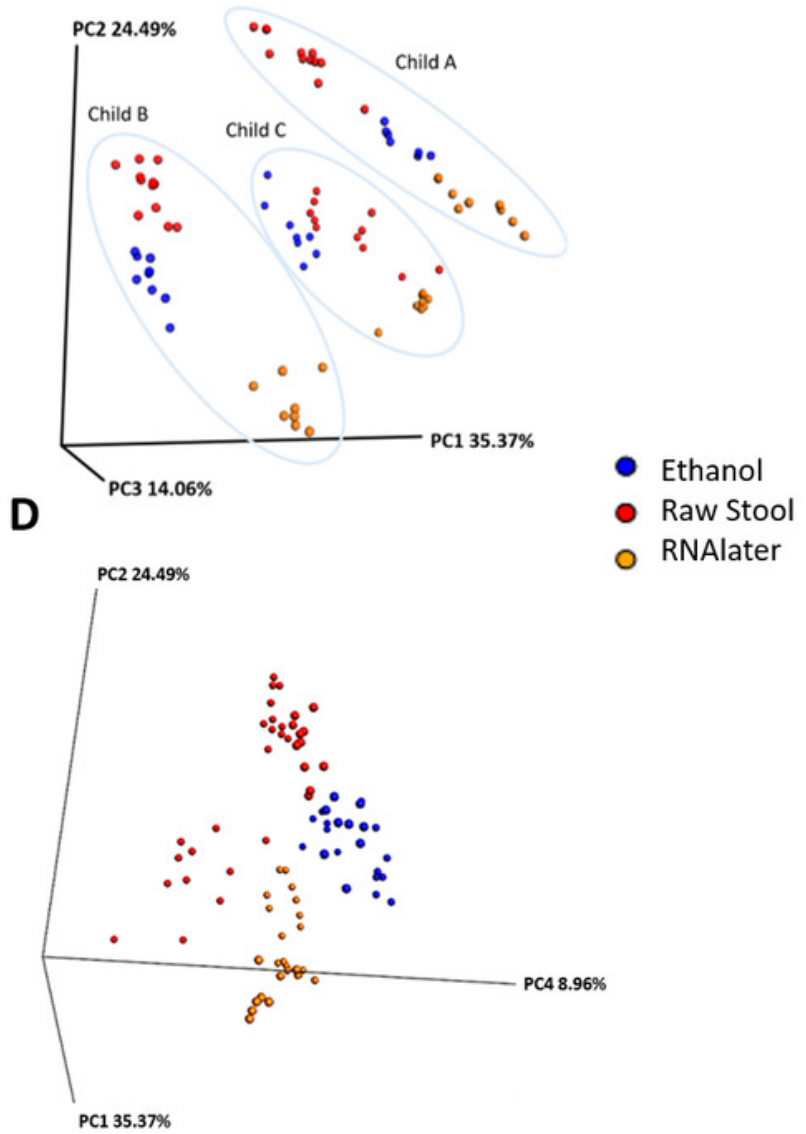


\section{Figure 2}

Relative bacterial abundance patterns of samples at the phyla (A) and genus (B) level varies between children and storage technique used within each child.

The top ten genera are included in the legend (B); where a genus name was not provided the lowest taxonomic resolution has been used where $p=$ phylum, $c=$ class, $0=$ order and $f=$ family. For a full annotation of the genus legend refer to the Supplemental Genus Legend. For a full description of sample codes refer to Table S1. Individual letter descriptors indicate the mean average relative abundances of raw stool (C), ethanol (E) and RNAlater (R) storage preservation across all children, time points and stool regions. 
A
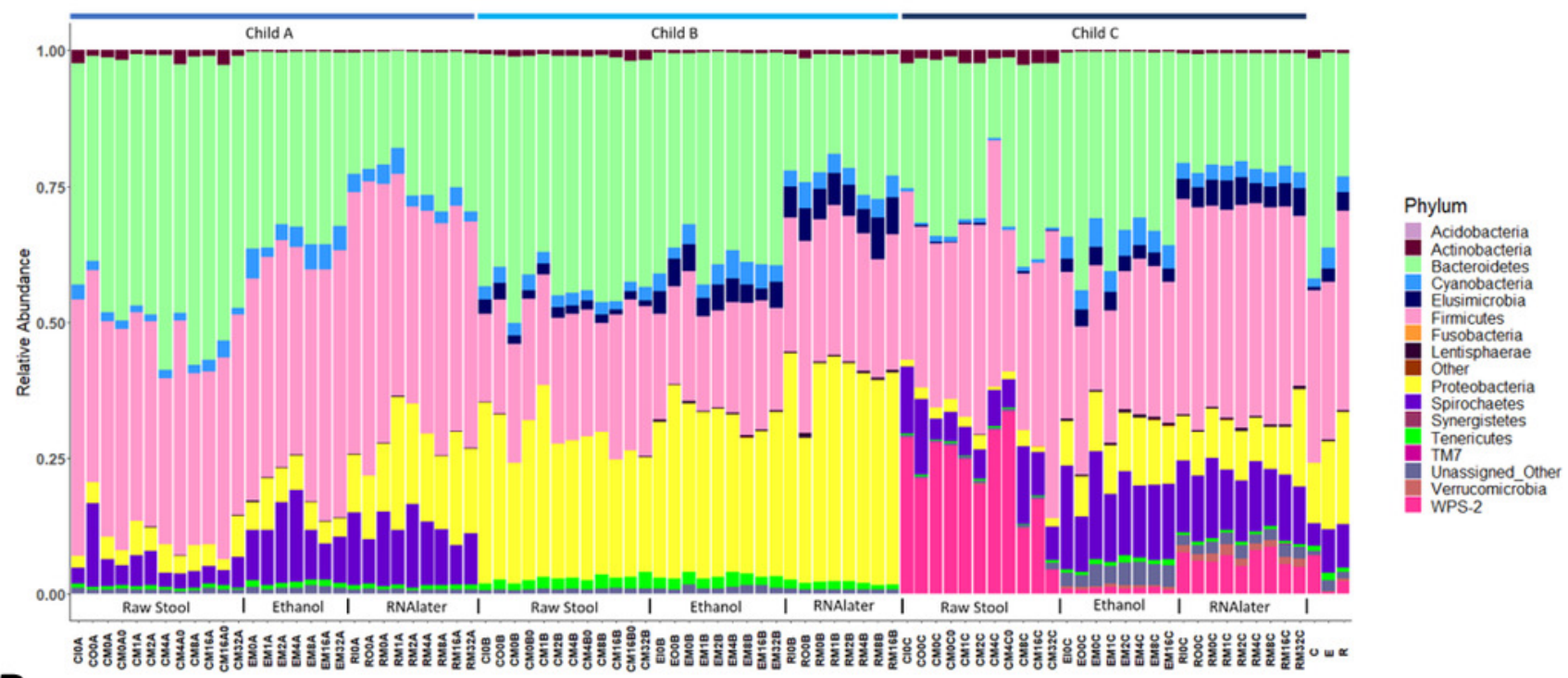

B

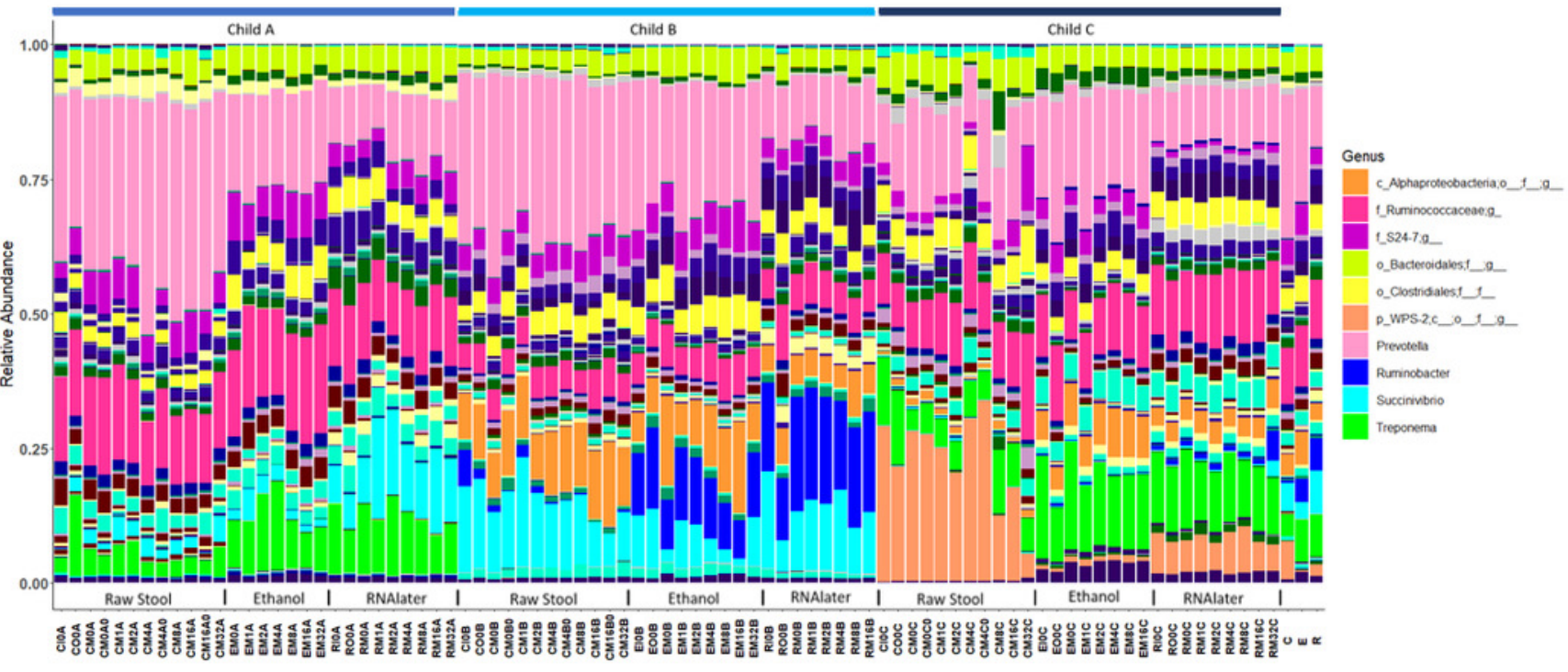




\section{Figure 3}

Bacterial groups significantly positively associated with different preservation methods of storage by linear discriminant analysis effect size (LEfSe).

Raw Stool = raw stool versus ethanol and RNAlater; Ethanol = ethanol versus raw stool only; and RNAlater $=$ RNAlater versus raw stool only. The significance parameters (LDA Score (log $10) \geq 2, p<0.01$ ) were met within each individual and when averaged across all three children to be included. The most descriptive taxonomic resolution is provided unless a higher taxonomy was more significant, in which case both are shown (For all information refer to Data S3).

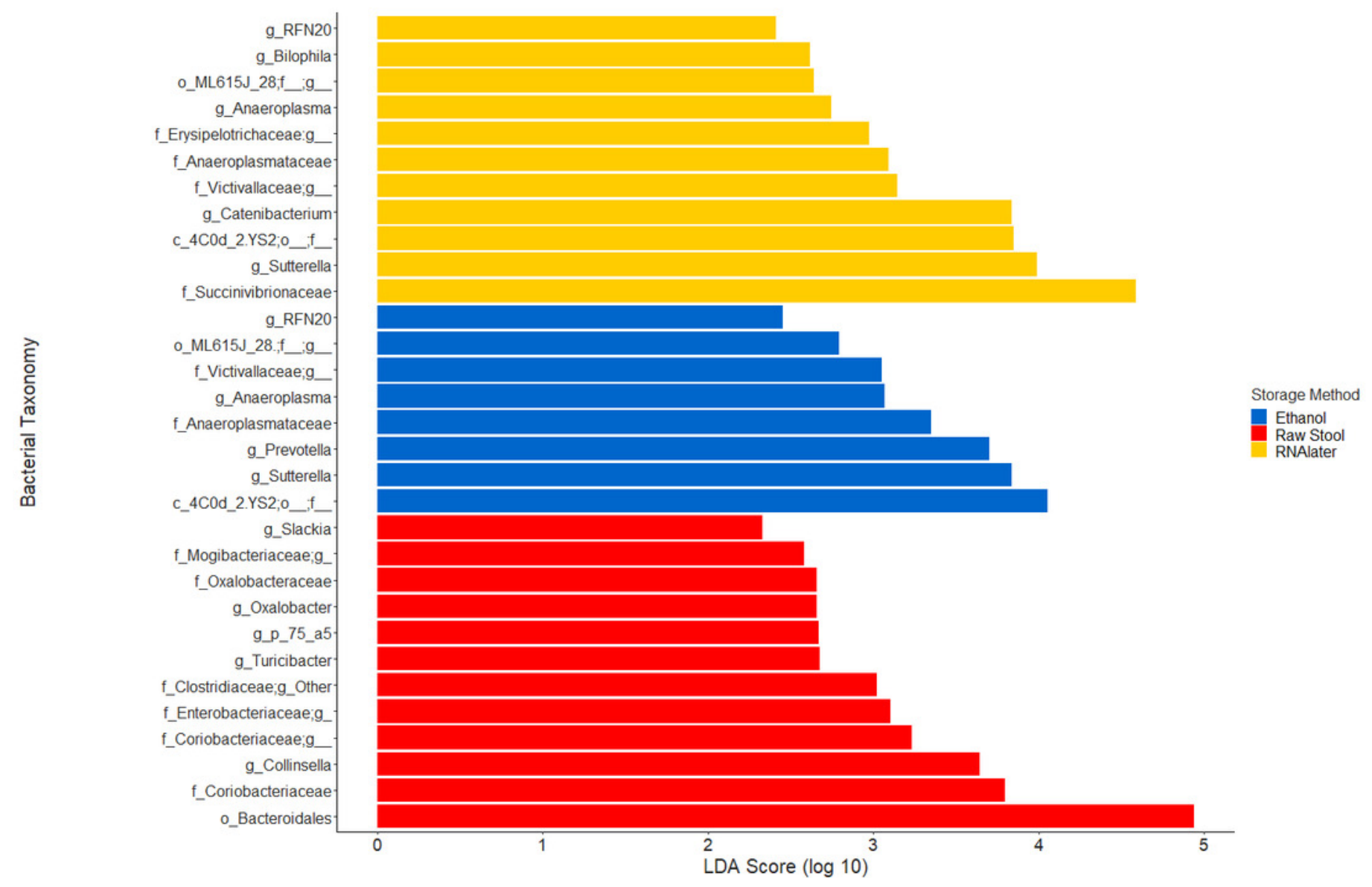




\section{Figure 4}

Microbiome relative abundance profiles remain relatively stable over time across all storage methods used at the phylum $(A-C)$ and genus $(D-F)$ levels.

The top ten genera are included in the legend $(B)$; where a genus name was not provided the lowest taxonomic resolution has been used where $p=$ phylum, $c=$ class, $0=$ order and $f=$ family. For a full annotation of the genus legend refer to the Supplemental Genus Legend. Raw stool ( $A$ and D), 100\% ethanol ( $B$ and $E$ ) and RNAlater ( $C$ and $F$ ). Samples were averaged across all three children and include all stool regions.
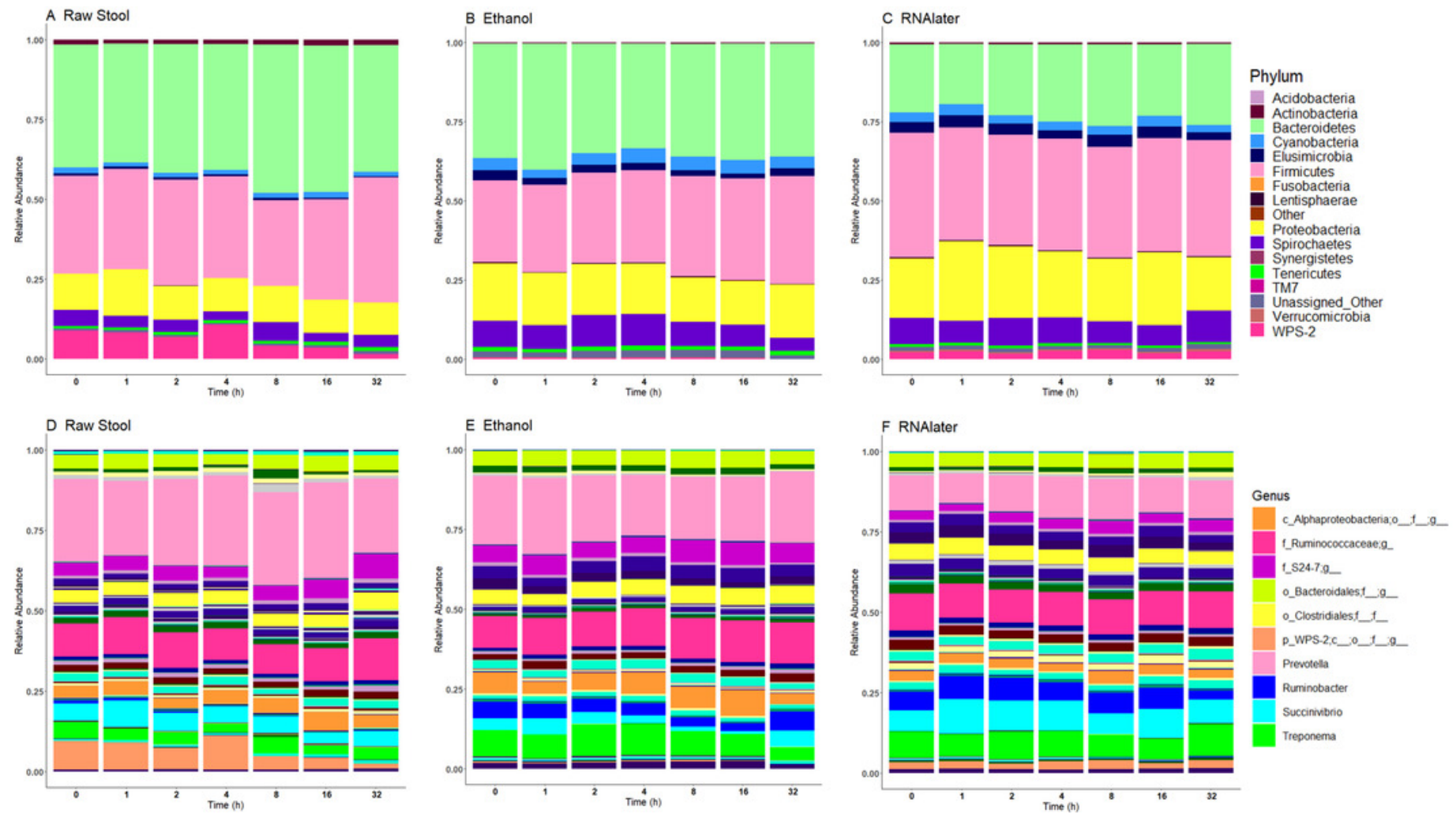
Figure 5

Figure 5: Bacterial groups identified to be significantly more abundant in raw stool samples at $32 \mathrm{~h}$ time-to-freezing by LEfSe analysis: Sediminibacterium (A) and Rummeliibacillus (B).

For samples to be included they must meet the following criteria: LDA Score $(\log 10) \geq 2, p<$ 0.01 .
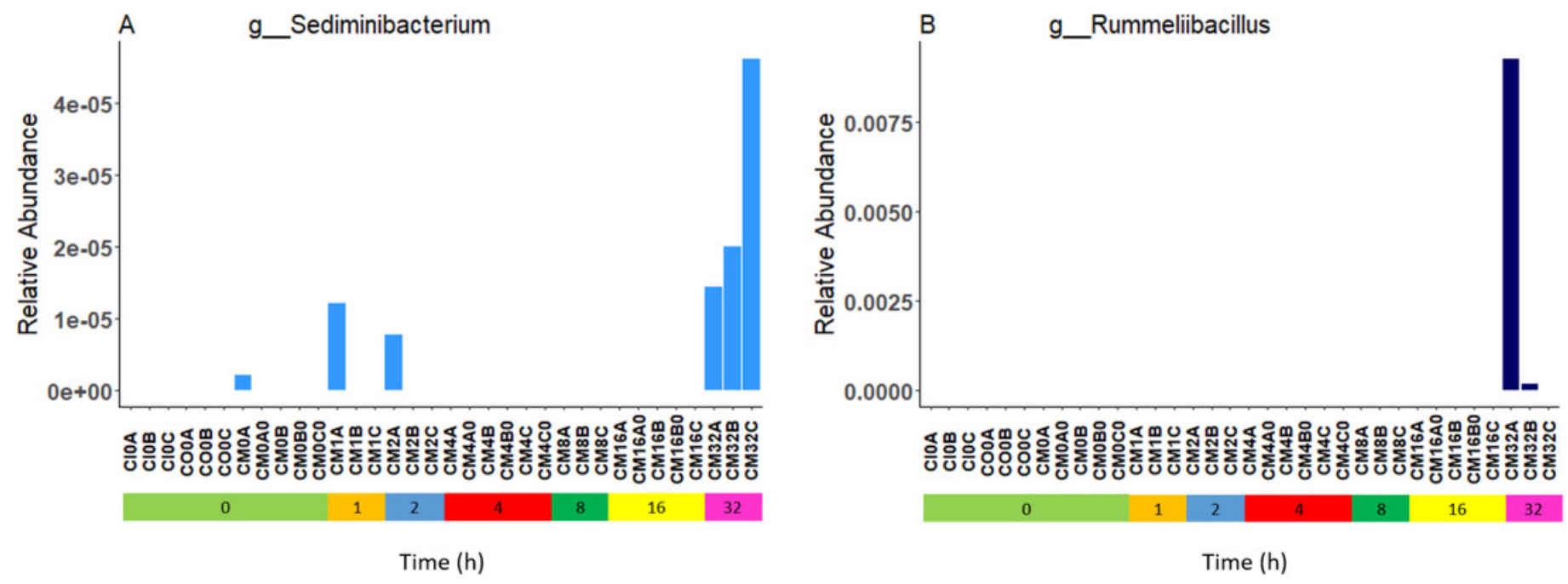


\section{Figure 6}

Microbiome profiles remained stable across stool regions.

Phyla (A) and genera (B). The top ten genera are included in the legend; where a genus name was not provided the lowest taxonomic resolution has been used where $p=$ phylum, $c$ $=$ class, $0=$ order and $f=$ family. For a full annotation of the genus legend refer to the Supplemental Genus Legend.
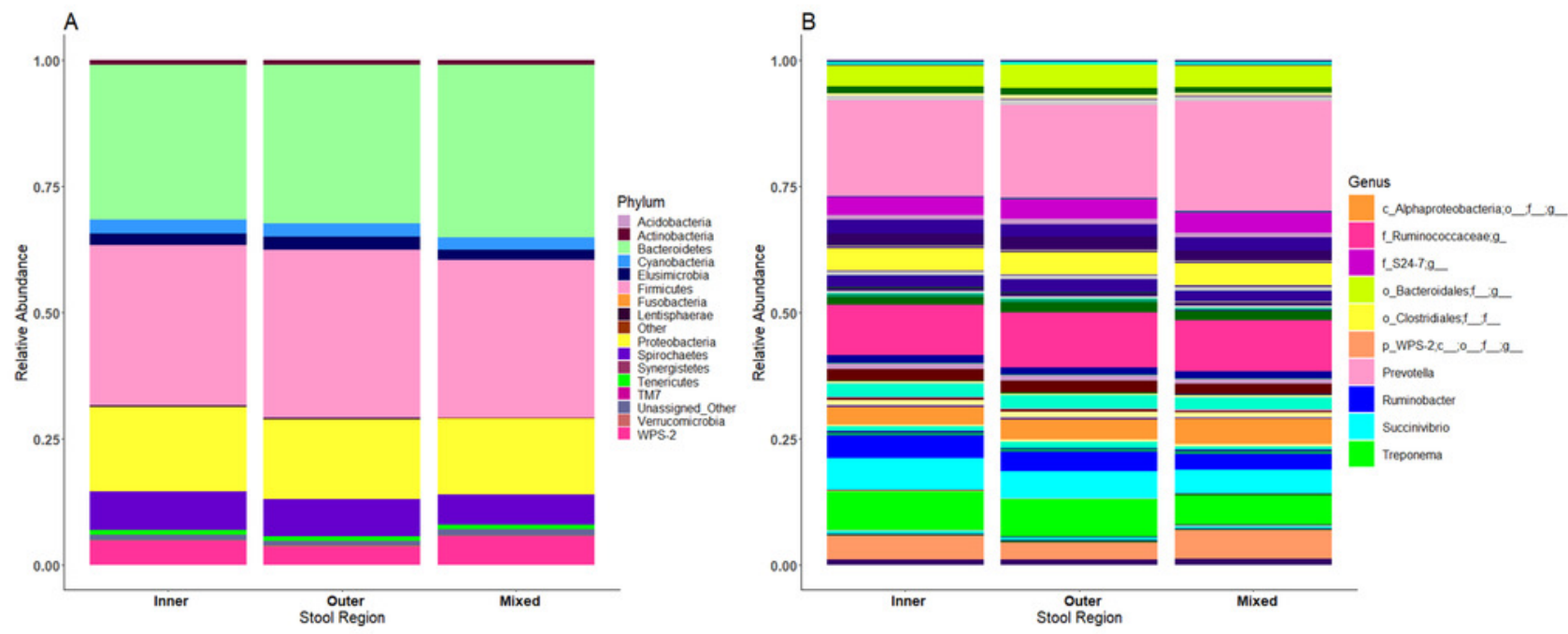